LETTER TO THE EDITOR

\title{
ALMA detects a radial disk wind in DG Tauri (Corrigendum)
}

\author{
M. Güdel ${ }^{1}$, C. Eibensteiner ${ }^{1}$, O. Dionatos ${ }^{1}$, M. Audard ${ }^{2}$, J. Forbrich ${ }^{3}$, S. Kraus ${ }^{4}$, Ch. Rab ${ }^{5}$, Ch. Schneider ${ }^{6}$, \\ S. Skinner ${ }^{7}$, and E. Vorobyov ${ }^{1,8}$ \\ 1 University of Vienna, Dept. of Astrophysics, Türkenschanzstr. 17, 1180 Vienna, Austria \\ e-mail: manuel.guedel@univie.ac.at \\ 2 Department of Astronomy, University of Geneva, Ch. d'Ecogia 16, 1290 Versoix, Switzerland \\ 3 University of Hertfordshire, Hatfield, Hertfordshire, UK \\ 4 University of Exeter, School of Physics and Astronomy, Stocker Road, Exeter EX4 4QL, UK \\ 5 Kapteyn Astronomical Institute, University of Groningen, PO Box 800, 9700 AV Groningen, The Netherlands \\ 6 Hamburger Sternwarte, Gojenbergsweg 112, 21029 Hamburg, Germany \\ 7 Center for Astrophysics and Space Astronomy, University of Colorado, 389 UCB, Boulder, CO 80309-0389, USA \\ 8 Research Institute of Physics, Southern Federal University, Stachki Ave. 194, Rostov-on-Don, Russia
}

A\&A, 620, L1 (2018), https://doi .org/10.1051/0004-6361/201834271

Key words. stars: pre-main sequence - stars: winds, outflows - protoplanetary disks - errata, addenda

Following a production error, many arcsecond signs $\left({ }^{\prime \prime}\right)$ were replaced with degree signs $\left({ }^{\circ}\right)$ in the original version of this paper. Here we republish the paper in its entirety, correcting the errors. 


\title{
ALMA detects a radial disk wind in DG Tauri ${ }^{\star}$
}

\author{
M. Güdel ${ }^{1}$, C. Eibensteiner ${ }^{1}$, O. Dionatos ${ }^{1}$, M. Audard ${ }^{2}$, J. Forbrich ${ }^{3}$, S. Kraus ${ }^{4}$, Ch. Rab ${ }^{5}$, Ch. Schneider ${ }^{6}$, \\ S. Skinner ${ }^{7}$, and E. Vorobyov ${ }^{1,8}$ \\ 1 University of Vienna, Dept. of Astrophysics, Türkenschanzstr. 17, 1180 Vienna, Austria \\ e-mail: manuel.guedel@univie.ac.at \\ 2 Department of Astronomy, University of Geneva, Ch. d'Ecogia 16, 1290 Versoix, Switzerland \\ 3 University of Hertfordshire, Hatfield, Hertfordshire, UK \\ ${ }^{4}$ University of Exeter, School of Physics and Astronomy, Stocker Road, Exeter EX4 4QL, UK \\ 5 Kapteyn Astronomical Institute, University of Groningen, PO Box 800, 9700 AV Groningen, The Netherlands \\ ${ }^{6}$ Hamburger Sternwarte, Gojenbergsweg 112, 21029 Hamburg, Germany \\ 7 Center for Astrophysics and Space Astronomy, University of Colorado, 389 UCB, Boulder, CO 80309-0389, USA \\ 8 Research Institute of Physics, Southern Federal University, Stachki Ave. 194, Rostov-on-Don, Russia
}

Received 18 September 2018 / Accepted 20 October 2018

\begin{abstract}
Aims. We aim to use the high spatial resolution of the Atacama Large Millimeter/submillimeter Array (ALMA) to map the flow pattern of molecular gas near DG Tauri and its disk, a young stellar object driving a jet and a molecular outflow.

Methods. We use observations from ALMA in the $J=2-1$ transition of ${ }^{12} \mathrm{CO},{ }^{13} \mathrm{CO}$, and $\mathrm{C}^{18} \mathrm{O}$ to study the Keplerian disk of DG Tauri and outflows that may be related to the disk and the jet.

Results. We find a new wind component flowing radially at a steep angle $\left(\approx 25^{\circ}\right.$ from the vertical $)$ above the disk with a velocity of $\approx 3.1 \mathrm{~km} \mathrm{~s}^{-1}$. It continues the trend of decreasing velocity for increasing distance from the jet axis ("onion-like velocity structure"). Conclusions. The new component is located close to the protostellar disk surface and may be related to photoevaporative winds.
\end{abstract}

Key words. stars: pre-main sequence - stars: winds, outflows - protoplanetary disks

\section{Introduction}

Protostellar outflows, jets, and disk winds play important roles in disk accretion, dispersal, and angular momentum transport (Frank et al. 2014). Observations indicate a physical link between accretion and "ejection", the mass outflow from a disk typically amounting to a few percent of the accretion rate onto the star (White \& Hillenbrand 2004). While collimated atomic jets reach velocities up to several $100 \mathrm{~km} \mathrm{~s}^{-1}$, molecular outflows are slower (up to a few tens of $\mathrm{km} \mathrm{s}^{-1}$ ) and less well collimated. They may result from entrainment by the fast jet interacting with the disk material and the surrounding protostellar envelope (Dionatos \& Güdel 2017). Slow disk winds may also result from magnetocentrifugal acceleration (with velocities of a few times the sound velocity at a few scale heights above the disk; Bai \& Stone 2013) and/or from X-ray/ultraviolet disk irradiation from the central star (Ercolano et al. 2008; Gorti \& Hollenbach 2009). Such photoevaporative winds are typically launched at a few tens of astronomical units from the star. Kitamura et al. (1996a; hereafter K96) proposed a process wherein a strong stellar wind interacts with the disk surface to drive a near-radial expansion and therefore erosion of the disk. In this Letter we present ALMA observations that trace disk mass loss in the young stellar object DG Tauri.

\footnotetext{
* The reduced datacubes are only available at the CDS via anonymous ftp to cdsarc.u-strasbg.fr $(130.79 .128 .5)$ or via http: //cdsarc.u-strasbg.fr/viz-bin/cat/J/A+A/620/L1
}

\section{The target: DG Tauri}

DG Tauri is, judging from its "flat" spectrum, at a transition between a Class-I protostar and a classical T Tauri star (Pyo et al. 2003; Calvet et al. 1994). It is surrounded by a disk of gas and dust (Dutrey et al. 1996; Kitamura et al. 1996a,b; Testi et al. 2002), with a size in the millimeter continuum of about 1". $1 \times 0$ 0".6 (133 au $\times 73 \mathrm{au}$; Dutrey et al. 1996). DG Tauri ejects a well studied collimated jet with velocities of several $100 \mathrm{~km} \mathrm{~s}^{-1}$ (e.g., Lavalley et al. 1997; Eislöffel \& Mundt 1998; White et al. 2014). We use a distance to DG Tauri of 121.2 (119.1-123.4) pc measured by Gaia (Gaia Collaboration 2016, 2018 ), a value much smaller than hitherto assumed $(d \approx 140 \mathrm{pc})$.

Kitamura et al. (1996a) mapped DG Tauri's large-scale environment in ${ }^{13} \mathrm{CO}(1-0)$, revealing molecular gas in an extended disk-like structure with a radius of $\sim 2800$ au (for $d=$ $140 \mathrm{pc}$ ) showing, instead of a rotational velocity pattern, radial expansion with a velocity of $\sim 1.5 \mathrm{~km} \mathrm{~s}^{-1}$. Testi et al. (2002) additionally mapped the Keplerian motion of the inner disk. Higher-excitation ${ }^{12} \mathrm{CO}(6-5)$ and ${ }^{12} \mathrm{CO}(3-2)$ observations presented earlier by Schuster et al. (1993) indicated a narrow line, cut off by blueshifted self absorption, plus extended wings (of a few $\mathrm{km} \mathrm{s}^{-1}$ ) indicating an outflow.

Takami et al. (2004) and Beck et al. (2008) identified a cone-like structure of outflowing gas surrounding the jet axis in near-infrared $\mathrm{H}_{2}$ emission, with a radius at its distant end of about $40 \mathrm{au}$ and a flow velocity of about $15 \mathrm{~km} \mathrm{~s}^{-1}$. Agra-Amboage et al. (2014) suggested a hollow-cavity geometry with a half-opening angle of $30^{\circ}$ and velocities 
down to a few kilometers per second at larger distances from the axis; the structure was furthermore observed in ultraviolet $\mathrm{H}_{2}$ fluorescence lines by Schneider et al. (2013) using the Hubble Space Telescope. Overall, a picture is emerging in which the jet/outflow/wind system shows an onion-like structure around the axis with decreasing velocity for increasing distance from the axis (Takami et al. 2004; Agra-Amboage et al. 2014).

\section{Observations}

Our observations of DG Tauri were obtained by the Atacama Large Millimeter/submillimeter Array (ALMA) in Cycle 3 on 24/26 September 2016 using 42 of the $12 \mathrm{~m}$ antennas, providing an angular resolution (beam size) of $\sim 0^{\prime \prime} .21 \times 00^{\prime \prime} 12$ $(25 \mathrm{au} \times 15 \mathrm{au})$. The observations in band 6 comprise the transitions of ${ }^{12} \mathrm{CO} J=2-1(230.538 \mathrm{GHz}),{ }^{13} \mathrm{CO} J=2-1$ $(220.399 \mathrm{GHz})$, and $\mathrm{C}^{18} \mathrm{O} J=2-1(219.560 \mathrm{GHz})$. We also included the $\mathrm{SiO} J=5-4(217.105 \mathrm{GHz})$ transition in the setup but did not obtain any detection. The spectral bandwidth was $117 \mathrm{MHz}$ (corresponding to a velocity range of $\sim 152-162 \mathrm{~km} \mathrm{~s}^{-1}$ per sideband), and the spectral resolution was $0.122 \mathrm{MHz}$ (velocity resolution of $\sim 0.159-0.167 \mathrm{~km} \mathrm{~s}^{-1}$ ). The on-source time amounted to $1.95 \mathrm{~h}$. Three extragalactic calibrators were used (quasars J0238+1636, J0510+1800, J0403+2600). We applied standard procedures provided by the CASA software version 4.7.0-1 for data reduction, calibration, and data extraction. The line data presented here were continuum-subtracted and cleaned following prescriptions in the CASA User Reference and Cookbook, public release 2017. Typical background noise levels were $3.8,4.3$, and $3.1 \mathrm{mJy}$ beam ${ }^{-1}$ for ${ }^{12} \mathrm{CO},{ }^{13} \mathrm{CO}$, and $\mathrm{C}^{18} \mathrm{O}$, respectively. Velocities are given relative to the local standard of rest (lsr) unless noted otherwise (Sect. 5)

\section{Results}

Figure 1 shows first-moment (flux-weighted velocity) maps with the color coding given in the bar on the right. The gas emission extends to larger radii than the dust continuum disk (overplotted in contours) as is typically observed (e.g., Ansdell et al. 2018; Facchini et al. 2017). The $\mathrm{C}^{18} \mathrm{O}$ (a) and ${ }^{13} \mathrm{CO}$ (b) maps indicate a symmetric disk compatible with an inclination of $\sim 38^{\circ}$ (Eislöffel \& Mundt 1998). The position-velocity diagram (Fig. 1c) extracted along the disk major axis of the ${ }^{13} \mathrm{CO}$ map agrees with a Kepler rotation profile for a star of 0.5 solar masses $\left(M_{\odot}\right.$; solid lines; similar for $\left.{ }^{18} \mathrm{CO}\right)$. These figures indicate a systemic velocity relative to $1 \mathrm{sr}$ of $v_{\text {sys }} \approx 5.5 \mathrm{~km} \mathrm{~s}^{-1}$, in agreement with Testi et al. (2002) and K96. Judging from the jet geometry (the approaching jet points toward a position angle of $\sim 222^{\circ}$, that is, to the southwest (SW), Lavalley et al. 1997), the southeast (SE) half of the disk is approaching, the disk rotates clockwise, and thus the northeast (NE) half-disk is in front. Around zero velocity relative to $v_{\text {sys }}$ (NE-SW diagonal) the ${ }^{13} \mathrm{CO}$ disk emission is subject to absorption, supporting reports by Schuster et al. (1993; line profile) and Testi et al. (2002; channel maps).

The ${ }^{12} \mathrm{CO}$ first-moment map, in contrast, reveals complex velocity structure presented separately for the blue- and redshifted channels in Fig. 2. The blueshifted emission (a) is concentrated toward SE-E-NE-N-NW of the center for $v<$ $3.5 \mathrm{~km} \mathrm{~s}^{-1}$, while redshifted emission (b) is seen almost everywhere for $v>7 \mathrm{~km} \mathrm{~s}^{-1}$. The $3.5-5.5 \mathrm{~km} \mathrm{~s}^{-1}$ range is almost completely absorbed probably by cool foreground material similar to the ${ }^{13} \mathrm{CO}$ map (Fig. 1b) except for the $4.5-4.8 \mathrm{~km} \mathrm{~s}^{-1}$ interval. The key features of the ${ }^{12} \mathrm{CO}$ maps are (labeled in Figs. A.1 and A.3) as follows.
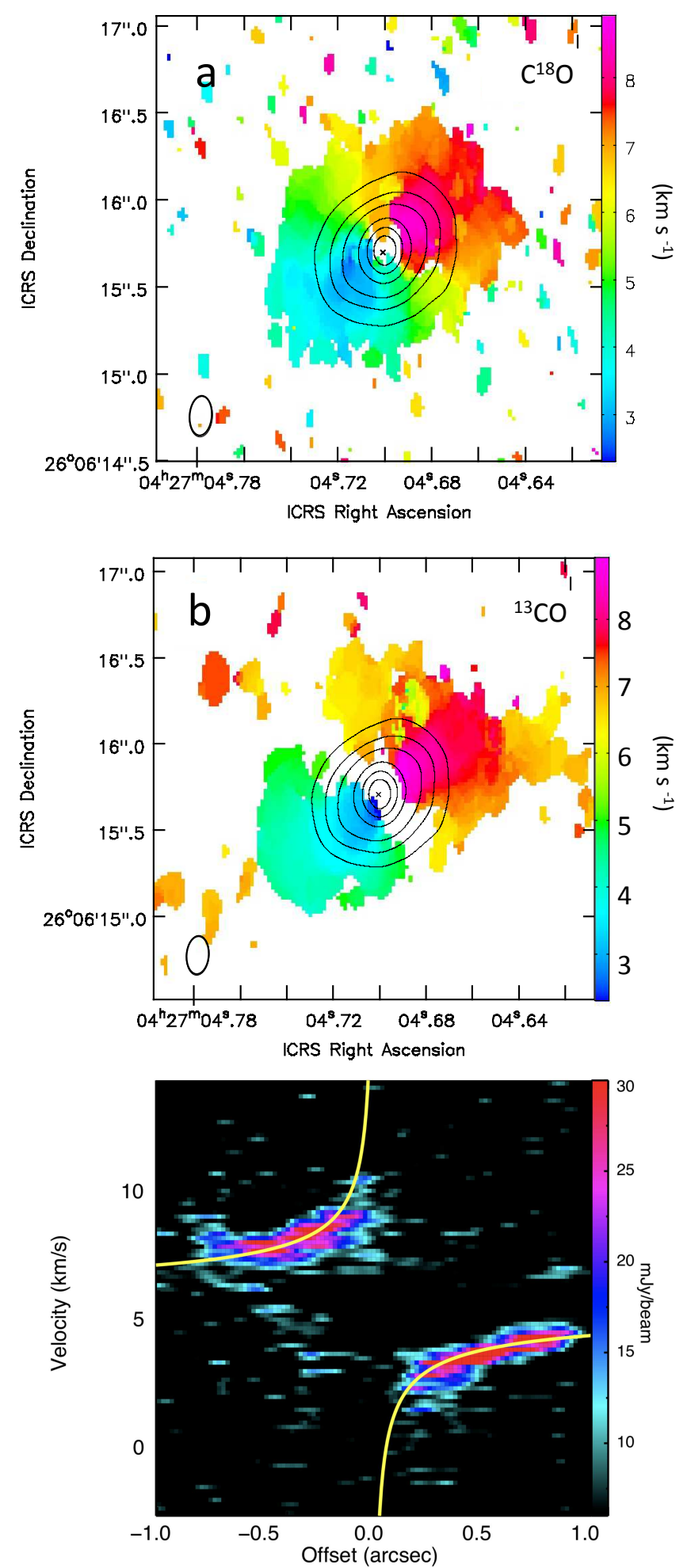

Fig. 1. Top and center panels: first-moment maps for $\mathrm{C}^{18} \mathrm{O}$ (top; $v_{\mathrm{lsr}}=2.3-8.9 \mathrm{~km} \mathrm{~s}^{-1}$ ) and ${ }^{13} \mathrm{CO}$ (middle; $v_{\mathrm{lsr}}=1.5-8.2 \mathrm{~km} \mathrm{~s}^{-1}$ ). Beam size is 0 ". $23 \times 0$ '.13 (ellipses at lower left). Contours mark the continuum emission levels of 9.4, 15.1, 20.8, 26.5, 37.8, 49.2 mJy beam $^{-1}$, with a peak at $60.6 \mathrm{mJy}$ beam $^{-1}$. The cross symbol marks the position of the star. Bottom panel: position-velocity diagram along major axis of projected disk in ${ }^{13} \mathrm{CO}$; position angle $134.3^{\circ}$. Yellow curves represent the Keplerian profiles for a $0.5 M_{\odot}$ star.

1. Within the projection of the continuum disk, the Keplerian disk is evident, especially near the disk center (strongly redshifted in the NW and blueshifted in the SE). 

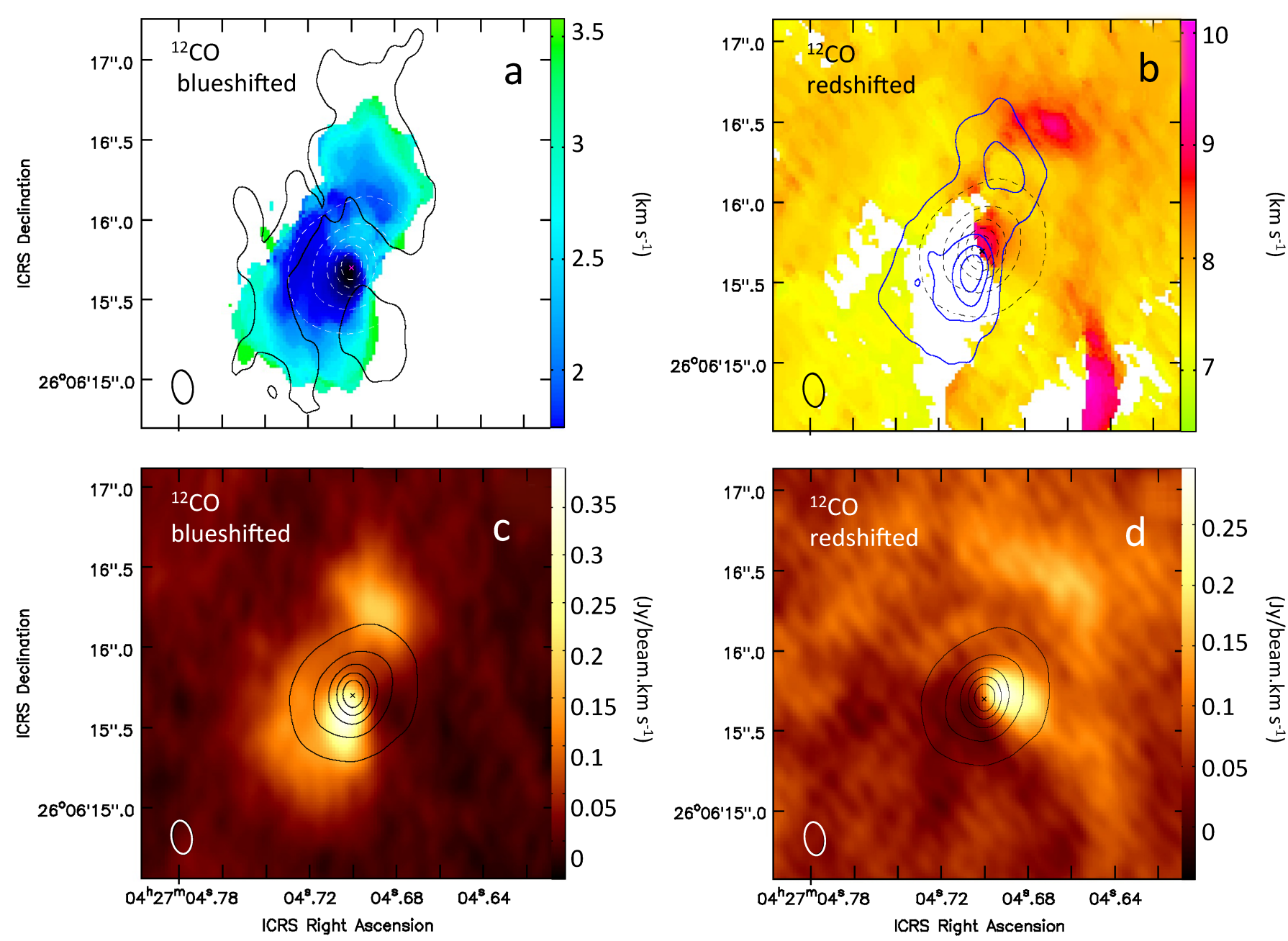

Fig. 2. Top row: ${ }^{12} \mathrm{CO}$ first-moment maps - Left: blueshifted, $v_{\mathrm{lsr}}=-1.2-+3.6 \mathrm{~km} \mathrm{~s}^{-1}$ (color; black below $1.6 \mathrm{~km} \mathrm{~s}^{-1}$ ) and $4.5-4.8 \mathrm{~km} \mathrm{~s}^{-1}($ solid outline). Dashed contours represent the continuum emission $\left(4.3,15.1,26.5,37.8,49.2\right.$ mJy beam $^{-1}$, peak $60.6 \mathrm{mJy}^{\text {beam }}{ }^{-1}$ ). The beam size is $0^{\prime \prime} .21 \times 00^{\prime \prime} .12$. Right: redshifted, $v_{\mathrm{lsr}}=7.0-10.1 \mathrm{~km} \mathrm{~s}^{-1}$. Solid contours represent blueshifted intensity from panel c. Dashed contours and beam size are as in panel a. Bottom row: intensity maps - Left: blueshifted intensity $\left(v_{\mathrm{lsr}}=-1.2-+4.8 \mathrm{~km} \mathrm{~s}^{-1}\right)$. Beam size is $0^{\prime \prime} 21 \times 0$ '. 12 . Background $\mathrm{rms} \approx 9.6 \mathrm{mJy}_{\text {beam }}^{-1} \mathrm{~km} \mathrm{~s}^{-1}$, peak flux $=0.39 \mathrm{Jy} \mathrm{beam}^{-1} \mathrm{~km} \mathrm{~s}^{-1}$. Right: redshifted intensity $\left(v_{\mathrm{lsr}}=+7.0-+11.4 \mathrm{~km} \mathrm{~s}^{-1}\right)$. Background $\mathrm{rms} \approx 12 \mathrm{mJy}_{\text {beam }} \mathrm{km} \mathrm{s}^{-1}$, peak flux $=0.28 \mathrm{Jy}_{\text {beam }}^{-1} \mathrm{~km} \mathrm{~s}^{-1}$. Beam size is as in panel c, solid contours in panels $c$ and $d$ show the same as the dashed contours in panels $a$ and $b$ ).

2. The Keplerian disk emission is asymmetric as bright redshifted and blueshifted intensity is missing toward $\mathrm{N}$ and $\mathrm{E}$, respectively.

3. Additional blueshifted emission appears toward the E and $\mathrm{N}$ (Fig 2a), the highest velocities apart from those in the disk center (dark blue) revealing an arc-like structure from SE to NW, approximately along the outermost two contours of the continuum disk (apart from additional blueshifted emission). The lowest-velocity blueshifted emission is seen in the 4.5$4.8 \mathrm{~km} \mathrm{~s}^{-1}$ window (solid contours in Fig. 2b) and reaches farther away from the star, also to SW-NW.

4. Much of the region outside the disk area shows redshifted emission, with two high-speed regions toward NW and SW (red, feature [5] in Fig. A.3).

\section{Discussion}

Feature [1] corresponds to the appearance of the first-moment map for ${ }^{13} \mathrm{CO}$ (Fig. 1b) where absorption suppresses emission from two wedge-shaped regions along the NE-SW diagonal, also seen for low velocities in ${ }^{12} \mathrm{CO}$ along the minor axis (see also Figs. 2c,d). Although parts of the outer disk can be traced out to $0{ }^{\prime \prime} .5-1^{\prime \prime}$, the Keplerian disk is very centrally brightened in a region with high radial velocities. However, in Figs. 2c,d both the redshifted and blueshifted high-velocity inner Keplerian disk emission regions are asymmetric [2] with respect to the major axis of the projected disk. The emission is almost completely absent in the NE half of the disk (which is closer to the observer). This feature is obviously related to the line of sight. We suggest that the specific viewing angles result in additional absorption; we emphasize that a wide range of (Keplerian) velocities $\left( \pm\right.$ several $\left.\mathrm{km} \mathrm{s}^{-1}\right)$ within a small area around the disk center are affected. We speculate that disk flaring leads to much longer line-of-sight paths through the local upper atmosphere on the closer half of the disk, perhaps leading to local "self-absorption" of the emission without velocity difference. The line-of-sight angle is less inclined with regard to the disk surface normal on the opposite side of the disk, meaning that the emission suffers less absorption. The flaring index of the relevant layer ( $\mathrm{CO}$ and/or dust) is not known, making a quantitative estimate challenging, but previously used indices of 1.2 and 1.25 (Guilloteau et al. 2011 and Podio et al. 2013, respectively) 


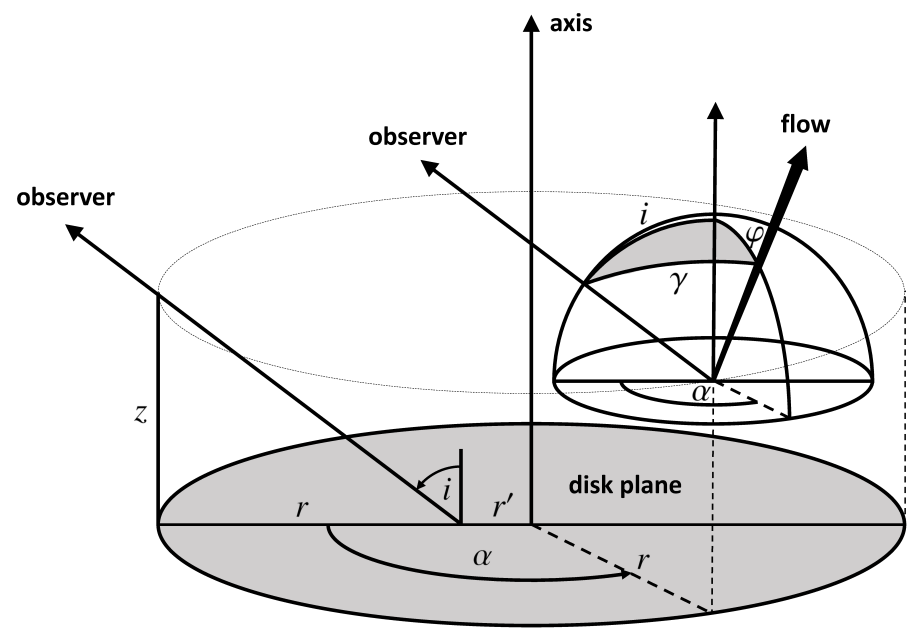

Fig. 3. Geometry of the flow relative to the disk and the line of sight (see main text and appendix for details).

give similar results and suggest a height of 10 au above the midplane at $r=69 \mathrm{au}$, roughly compatible with our estimate for the height of the wind structure derived below. Disk flaring could thus be a significant factor for absorption.

Feature [3] is the most important finding of these observations, revealing a mass flow that is not compatible with disk rotation but appears to flow radially outward above the disk surface. This material must be located in front of the disk.

We estimate the height of the emitting structure and its flow direction using Fig. 3 (see also Appendix C), adopting a disk inclination of $i=38^{\circ}$ against the line of sight (Eislöffel \& Mundt 1998). We henceforth use velocity offsets relative to the systemic velocity $v_{\text {sys }}=5.5 \mathrm{~km} \mathrm{~s}^{-1}$ and define an azimuth angle $\alpha$ measured on the disk clockwise from the NE minor axis. In Fig. 2a, the blueshifted arc is nearly concentric to the continuum contours. The distance of the fast flow $\left(v \lesssim-3 \mathrm{~km} \mathrm{~s}^{-1}\right)$ from the disk center along the major axis is $\approx 0^{\prime \prime} .45 \pm 00^{\prime \prime} .12$. If the arc structure is located at a height $z$ above the disk plane, then the line of sight through the arc at $\alpha=0^{\circ}$ cuts the disk plane on the minor axis at a distance of $r^{\prime}=r-z \tan i$ from the disk center. Projection owing to the disk inclination compresses $r^{\prime}$ to $r_{\text {proj }}^{\prime}=r^{\prime} \cos i=r \cos i-z \sin i$. We estimate $r_{\text {proj }}^{\prime}=00^{\prime \prime} .23-0^{\prime \prime} .44$. From

$$
\frac{z}{r}=\frac{\cos i-r_{\text {proj }}^{\prime} / r}{\sin i},
$$

and the requirement $z \geq 0$, we find $z / r=0.03-0.15$, that is, the flow emission is located immediately above the disk midplane ( $z \lesssim 10$ au for $r=69$ au [0'.57]).

The flow is detected only in the NE half of the disk (around $\alpha \approx 0^{\circ} \pm 90^{\circ}$ ), indicating that it is inclined outwards in such a way that the radial velocity at larger $\alpha$ is small enough for the flow to be subject to foreground absorption. We assume a flow velocity of $v$ at an angle of $\varphi$ to the local vertical on the disk midplane (Fig. 3), away from the axis. At an azimuth $\alpha$, the spherical triangle between the local vertical, the line of sight, and the flow direction (at angle $\varphi$ to the vertical in azimuth direction $\alpha$ ) requires that for the angle $\gamma$ between the flow and the line of sight,

$\cos \gamma=\cos i \cos \varphi+\sin i \sin \varphi \cos \alpha$,

and the radial flow velocity is $v_{\text {rad }}=v \cos \gamma$. From Fig. 2a, at the nearest point on the minor axis, with azimuth $\alpha_{1}=0$, the radial velocity is $v_{\text {rad }}\left(\alpha_{1}\right) \approx-3 \mathrm{~km} \mathrm{~s}^{-1}$. We estimate that for $\alpha_{2}=105^{\circ}$ (toward NW), the radial flow velocity reaches $v_{\text {rad }}\left(\alpha_{2}\right)=-2 \mathrm{~km} \mathrm{~s}^{-1}$ (and is absorbed at larger $\alpha$ and presumably smaller velocity offsets); the ratio $v_{\text {rad }}\left(\alpha_{2}\right) / v_{\text {rad }}\left(\alpha_{1}\right)=2 / 3$ determines, through Eq. (2) for the numerator and denominator, the value of $\varphi$. We obtain $\varphi \approx 25^{\circ}$. The flow close to the disk is therefore relatively steep; this geometry $(\varphi<i)$ explains why the fast blueshifted flow does not expand much to the NE. The full velocity is about $v=v_{\text {rad }}\left(\alpha_{1}\right) / \cos (\varphi-i) \approx-3 / \cos \left(-13^{\circ}\right)=$ $-3.1 \mathrm{~km} \mathrm{~s}^{-1}$. At an azimuth of $\alpha_{3}=180^{\circ}$, we expect a radial flow velocity of $v_{\text {rad }}\left(\alpha_{3}\right)=v \cos (\varphi+i) \approx-1.4 \mathrm{~km} \mathrm{~s}^{-1}$ relative to $v_{\text {sys }}$, that is, $4.1 \mathrm{~km} \mathrm{~s}^{-1}$ relative to $1 \mathrm{sr}$, which lies in the middle of the fully absorbed spectral region.

One possibility for the physical origin of this wind is photoevaporation driven by ultraviolet, extreme ultraviolet, or X-ray irradiation from the central star. Both the velocity and location of the feature agree with predictions for photoevaporative winds while all known magnetocentrifugal wind features known in DG Tauri are at significantly higher velocities and are much closer to the star. Predictions for observable features of photoevaporative winds concentrated on atomic or ionized species and it is difficult to estimate if heating is sufficient to lift the deeper disk layers where $\mathrm{CO}$ molecules abound. We used the resulting temperature structure from a thermochemical disk model of DG Tauri published by Podio et al. (2013) that considers the highenergy radiation of DG Tauri. We then determined the layer at which the escape temperature is reached using DG Tauri's highenergy luminosity (where $T_{\text {esc }}=G m_{\mathrm{H}} M_{*} /[k r], G=$ gravitational constant, $m_{\mathrm{H}}=$ mass of hydrogen atom, $M_{*}=$ stellar mass, $k=$ Boltzmann constant, $r=$ distance from star; Ercolano et al. 2008). This rough model predicts escape at $r=60-70$ au significantly above the molecular layer, but the exact model strongly depends on details such as the spectrum of the irradiation, the disk structure (e.g., flaring), and disk density. This requires a dedicated thermochemical model considering the new spatially resolved data (i.e., $\mathrm{C}^{18} \mathrm{O}$ for the disk structure).

The widely distributed redshifted emission [4] was already noted by Testi et al. (2002) and is probably related to the molecular gas in the larger region around DG Tauri. We note that the redshifted ${ }^{12} \mathrm{CO}$ line remains, expectedly, unaffected by the blueshifted flow in front, given the frequency shift of the line. Specifically, we note a strongly redshifted arc-like feature ([5] in Fig. A.3) approximately $1^{\prime \prime} \mathrm{SW}$ of the disk with a maximum radial velocity of about $+4.5 \mathrm{~km} \mathrm{~s}^{-1}$ (relative to $v_{\mathrm{sys}}$; Fig. 2b). This feature continues down to the central disk region at decreasing radial velocity (Fig. 2d). We speculate that this is an accretion flow from the remnant envelope in front of the disk spiraling toward the disk, similar to streams detected by ALMA in the protostar L1489 IRS (Yen \& Takakuwa 2014); the variation in redshift would then be due to projection. Another detached redshifted feature is seen to the $\mathrm{N}$.

\section{Conclusions}

Our ALMA observations uncover a new outflow component in DG Tauri. Our geometric estimates show that the wind may reach a velocity of about $3.1 \mathrm{~km} \mathrm{~s}^{-1}$, to be compared with $\sim 1.5 \mathrm{~km} \mathrm{~s}^{-1}$ at the larger distances reported by K96, supporting the picture of an "onion-like" structure of flows with a velocity gradient increasing toward the axis. The wind emission originates close to the disk surface at a disk radius of approximately 40-70 au and is inclined outward by about $25^{\circ}$ from the vertical. The size of this structure is much larger than the previously described $\mathrm{H}_{2}$ cone (e.g., Takami et al. 2004; Schneider et al. 2013). 
We speculate that we see $\mathrm{CO}$ gas entrained by a photoevaporative flow (Ercolano et al. 2008; Gorti \& Hollenbach 2009) driven outward either by inclined magnetic fields (for weakly ionized gas) or the action of the stellar wind. For large distances (50-100 au) in the early disk stages, far-ultraviolet-driven photoevaporation is particularly attractive (Gorti \& Hollenbach 2009) although model calculations have so far favored flows of atomic gas. Whether or not the flow we are observing here is related to the radial flow reported by $\mathrm{K} 96$ for much larger distances remains unclear.

Acknowledgements. SK acknowledges support from ERC grant No. 639889. EV acknowledges support from the Russian Ministry of Education and Science project 3.5602.2017. This paper makes use of the following ALMA data: ADS/JAO.ALMA\#2015.1.00722.S. ALMA is a partnership of ESO (representing its member states), NSF (USA) and NINS (Japan), together with NRC (Canada), NSC and ASIAA (Taiwan), and KASI (Republic of Korea), in cooperation with the Republic of Chile. The Joint ALMA Observatory is operated by ESO, AUI/NRAO and NAOJ. This work has made use of data from the European Space Agency (ESA) mission Gaia (https://www . cosmos.esa.int/gaia), processed by the Gaia Data Processing and Analysis Consortium (DPAC, https://www.cosmos.esa.int/web/gaia/dpac/ consortium). Funding for the DPAC has been provided by national institutions, in particular the institutions participating in the Gaia Multilateral Agreement.

\section{References}

Agra-Amboage, V., Cabrit, S., Dougados, C., et al. 2014, A\&A, 564, A11 Ansdell, M., Williams, J. P., Trapman, L., et al. 2018, ApJ, 859, 21 Bai, X.-N., \& Stone, J. M. 2013, ApJ, 769, 76

Beck, T. L., McGregor, P. J., Takami, M., \& Pyo, T.-S. 2008, ApJ, 676, 472

Boehler, Y., Weaver, E., Isella, A., et al. 2017, ApJ, 840, 60
Calvet, N., Hartmann, L., Kenyon, S. J., \& Whitney, B. A. 1994, ApJ, 434, 330 Cleeves, L. I., Öberg, K. I., Wilner, D. J., et al. 2014, ApJ, 832, 110

Dionatos, O., \& Güdel, M. 2017, A\&A, 579, A64

Dougados, C., Cabrit, S., Lavalley, C., \& Ménard, F. 2000, A\&A, 357, L61

Dunham, M. M., Vorobyov, E. I., \& Arce, H. G. 2014, MNRAS, 444, 887

Dutrey, A., Guilloteau, S., Duvert, G., et al. 1996, A\&A, 309, 493

Eislöffel, J., \& Mundt, R. 1998, AJ, 115, 1554

Ercolano, B., Drake, J. J., Raymond, J. C., \& Clarke, C. C. 2008, ApJ, 688, 398 Facchini, S., Birnstiel, T., Bruderer, S., \& van Dishoeck, E. F. 2017, A\&A, 605, A16

Frank, A., Ray, T. P., \& Cabrit, S., et al. 2014, in Protostars and Planets VI, eds. H. Beuther, R. S. Klessen, C. P. Dullemond, et al. (Tucson: University of Arizona Press), 451

Gaia Collaboration (Prusti, T., et al.) 2016, A\&A, 595, A1

Gaia Collaboration (Brown, A. G. A., et al.) 2018, A\&A, 616, A1

Gorti, U., \& Hollenbach, D. 2009, ApJ, 690, 1539

Guilloteau, S., Dutrey, A., Piétu, V., \& Boehler, Y. 2011, A\&A, 529, A105

Herczeg, G. J., Najita, J. R., Hillenbrand, L. A., \& Pascucci, I. 2007, ApJ., 607, 509

Kitamura, Y., Kawabe, R., \& Saito, M. 1996a, ApJ, 457, 277

Kitamura, Y., Kawabe, R., \& Saito, M. 1996b, ApJ, 465, L137

Lavalley, C., Cabrit, S., Dougados, C., Ferruit, P., \& Bacon, R. 1997, A\&A, 327, 671

Podio, L., Kamp, I., Codella, C., et al. 2013, ApJ, 766, L5

Pyo, T.-S., Kobayashi, N., Hayashi, M., et al. 2003, ApJ, 590, 340

Schneider, P. C., Eislöffel, J., Güdel, M., et al. 2013, A\&A, 557, A110

Schuster, K. F., Harris, A. I., Anderson, N., \& Russell, A. P. G. 1993, ApJ, 412, L67

Takami, M., Chrysostomou, A., Ray, T. P., et al. 2004, A\&A, 416, 213

Testi, L., Bacciotti, F., Sargent, A. I., Ray, T. P., \& Eislöffel, J. 2002, A\&A, 349, L31

White, R. J., \& Hillenbrand, L. A. 2004, ApJ, 616, 998

White, M. C., McGregor, P. J., Bicknell, G. V., Salmeron, R., \& Beck, T. L. 2014, MNRAS, 441, 1681

Yen, H.-W., Takakuwa, S., Ohashi, N., et al. 2014, ApJ, 793, 1 


\section{Appendix A: First-moment maps for ${ }^{12} \mathrm{CO}$}
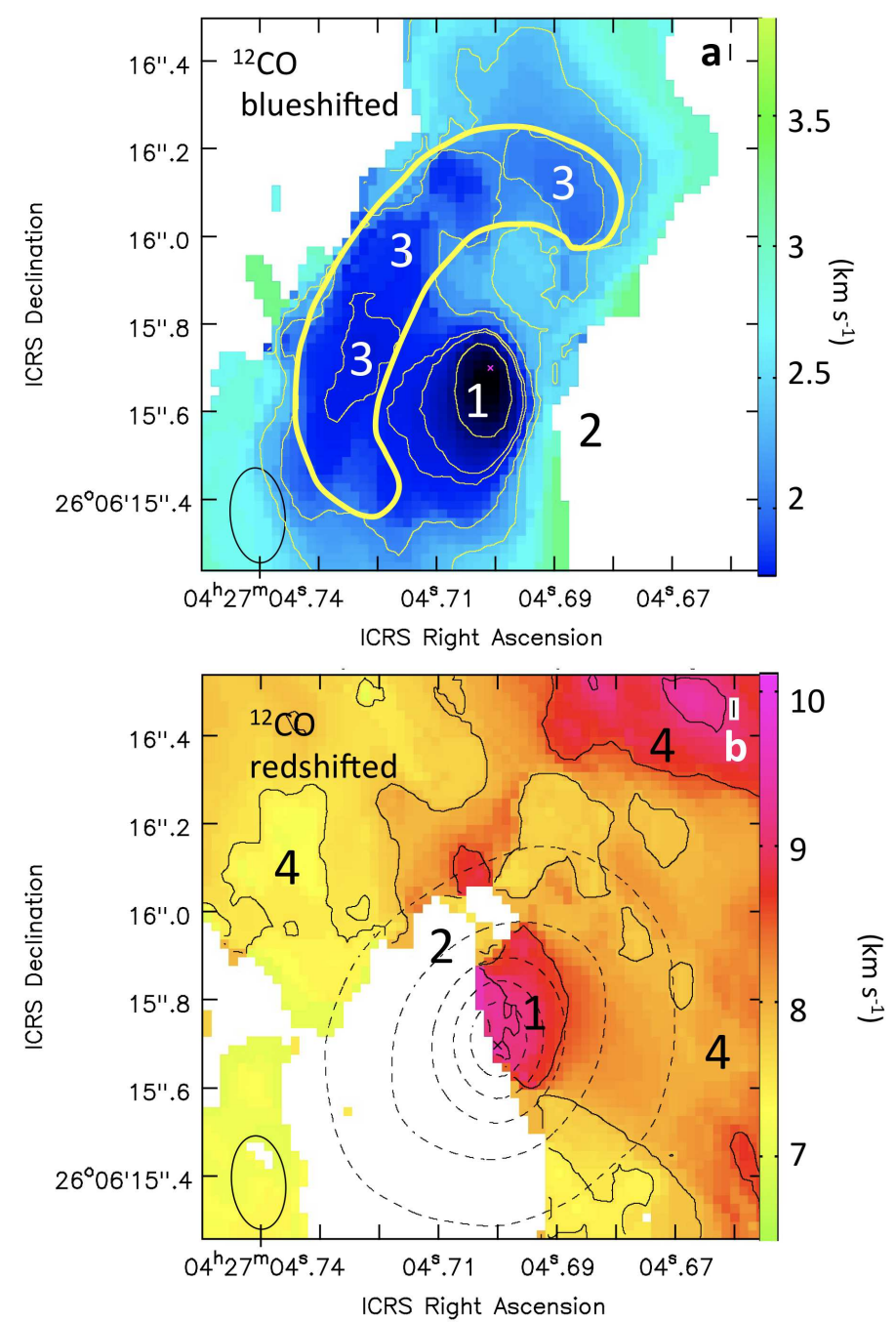

Fig. A.1. Zoom-in on the area of the continuum disk in Figs. 2a,b for ${ }^{12} \mathrm{CO}$. Labels $1-4$ refer to the features defined in Sect. 4. Top panel: blueshifted, $v_{\mathrm{lsr}}=-1.2-+3.6 \mathrm{~km} \mathrm{~s}^{-1}$ (color; black below $1.6 \mathrm{~km} \mathrm{~s}^{-1}$ ). Thin yellow contours are plotted for a few arbitrary velocity levels for visualization. The thick yellow outline marks the approximate area of the high-velocity wind. Bottom panel: redshifted, $v_{\mathrm{lsr}}=$ $7.0-10.1 \mathrm{~km} \mathrm{~s}^{-1}$. Thin black contours are overplotted for a few arbitrary velocity levels. Beam size: $00^{\prime \prime} 21 \times 00^{\prime \prime} 12$.

We show here more details related to Figs. 2a,b. Figure A.1 zooms in on the innermost parts, about coincident with the continuum-emitting dust disk (dashed contours in Fig. A.1b). We label here features [1]-[4] as defined in Sect. 4, and in particular outline the blueshifted arc-like structure [3] with a yellow line in Fig. A.1a.

In Fig. A.2 we show the combined full first moment map for ${ }^{12} \mathrm{CO}$ in a similar fashion as done in Figs. 1a,b for ${ }^{13} \mathrm{CO}$ and $\mathrm{C}^{18} \mathrm{O}$. While this figure shows the general arrangement of redand blueshifted kinematic features, we caution that independent blue- and redshifted features are superimposed along some lines of sight. For example, arrow "A" points to the inner Keplerian disk from which we measure, expectedly, redshifted emission. But Figs. 2a and A.1a show that there is also a blueshifted component along the same lines of sight, probably part of the wind. Arrow "B" points to a region that emits strongly blueshifted in

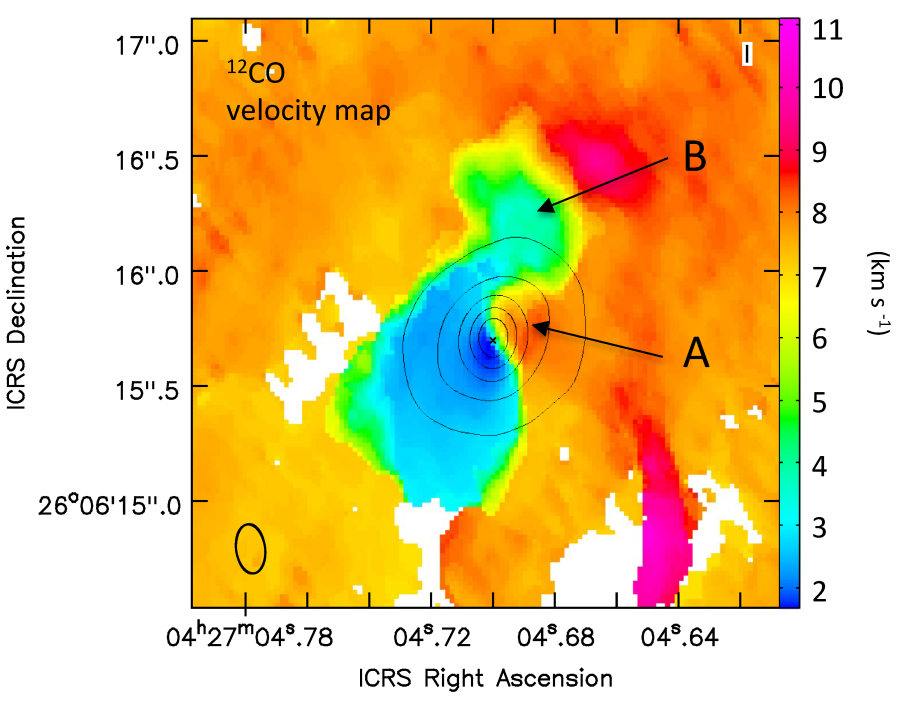

Fig. A.2. First-moment map for ${ }^{12} \mathrm{CO}\left(v_{\mathrm{lsr}}=1.5-11 \mathrm{~km} \mathrm{~s}^{-1}\right)$. Beam size: $0 \prime: 21 \times 0$ ". 12 (ellipse at lower left). Contours show continuum emission $\left(4.3,15.1,26.5,37.8,49.2 \mathrm{mJy}_{\text {beam }}^{-1}\right.$, peak $\left.60.6 \mathrm{mJy} \mathrm{beam}^{-1}\right)$. The cross symbol indicates the position of the star. Arrows point to problematic areas for this map (see text).

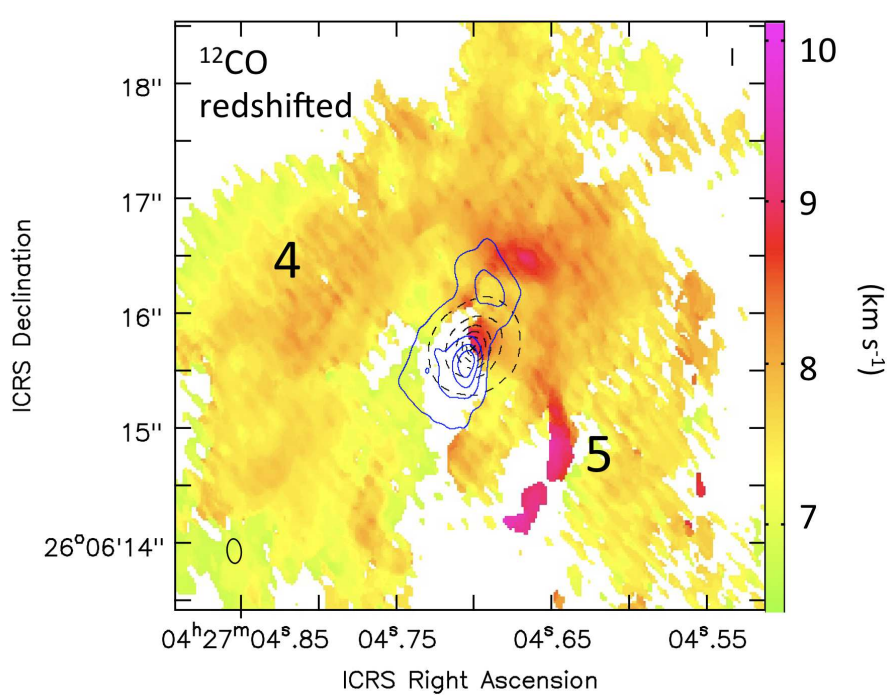

Fig. A.3. Larger field around DG Tauri in redshifted channels similar to Fig. 2b. We note the arc-like structure [5] at high velocity.

Figs. 2a and A.1a but is displayed here at a systemic velocity close to rest (green color), as there is also strong redshifted emission in the same region.

In Fig. A.3 we show the larger field around Fig. 2b, that is, the redshifted channels. In particular, the figure shows the arclike high-velocity structure in the SW [5] that is briefly described in Sect. 5. There are no significant blueshifted sources outside the field of Fig. 2a.

\section{Appendix $\mathrm{B}: \mathrm{C}^{18} \mathrm{O}$ and ${ }^{13} \mathrm{CO}$ intensity maps}

For completeness, we provide here some complementary observational information related to the intensity maps for $\mathrm{C}^{18} \mathrm{O}$ and ${ }^{13} \mathrm{CO}$; those of ${ }^{12} \mathrm{CO}$ were shown in the main paper (Figs. $2 \mathrm{c}, \mathrm{d}$ ). The $\mathrm{C}^{18} \mathrm{O}$ intensity map integrated over all relevant velocities (Fig. B.1-top) shows an ellipsoidal ring of emission between 

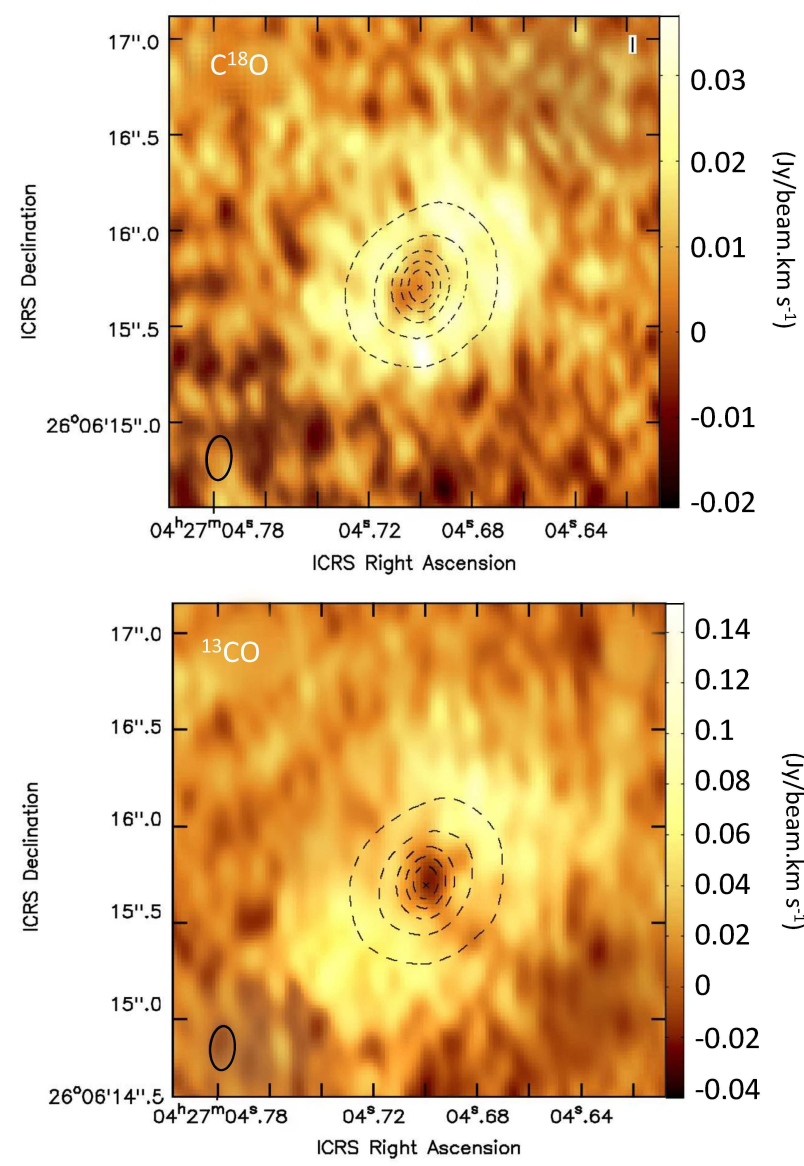

Fig. B.1. Intensity maps for $\mathrm{C}^{18} \mathrm{O}$ (top panel) and ${ }^{13} \mathrm{CO}$ (bottom panel) integrated over the entire velocity range of the line. We note the weak emission from the central regions and the diagonal (NE-SW) absorption band in the ${ }^{13} \mathrm{CO}$ map. Dashed contours represent the same as those in Figs. 2a,b.

radii of $\approx 0,2$ and 0 "'6 along the major axis. The emission inside $0 \prime \prime 2$ is faint. There is no indication for a velocity-specific absorption feature (see Fig. 1a). Apart from a real "hole" in the disk with depleted gas and therefore CO deficiency, various opacity effects could be acting. First, the dust could be optically thick in the inner disk, suppressing line emission (e.g., Cleeves et al. 2014 for IM Lup). For a derivation, one needs observations across a wide range of frequencies, which are presently not available in the same spatially resolved quality as used in our paper. Guilloteau et al. (2011) modeled the radial dependence of the dust opacity of DG Tauri using IRAM Plateau de Bure Interferometer data with a resolution down to $\sim 0$ ". 4 at $1.3-3 \mathrm{~mm}$ wavelengths; they found that the disk is optically thick within 40-50 au, compatible with the holes in our observations that reach out to at least $\sim 25 \mathrm{au}$. Numerical disk formation models indicate optically thick dust disks within about 10 au (Fig. 5 in Dunham et al. 2014).

On the other hand, the gas could be partially optically thick and absorb some dust continuum emission. Boehler et al. (2017) argued, for a similar observation of HD 142527, that the line emission is underestimated where the dust is bright and the dust emission is overestimated, because at the line frequency molecular gas can partially absorb dust emission. This leads to suppressed emission from the central region after subtraction of a constant background evaluated outside the line region in the

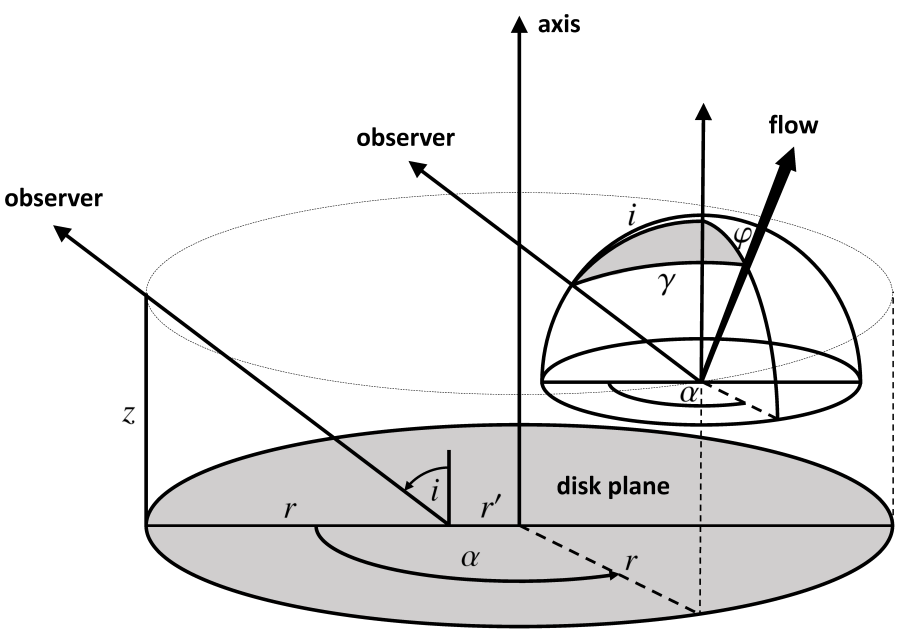

Fig. C.1. Geometry of the flow relative to the disk and the line of sight. The protostellar disk midplane is shaded in gray, out to a radius $r$. The line of sight is from the upper left; it is inclined by an angle $i$ to the disk axis. On the left side, a source at height $z$ above the disk is projected inward to a radius of $r^{\prime}$, which itself will be compressed to $r^{\prime} \cos i$ due to disk inclination $i$ (see Eq. (1)). The half-sphere on the right shows, for a point at projected disk radius $r$ at a height $z$ and azimuth angle $\alpha$, the relative geometry between the vertical (parallel to the disk axis), the line of sight to the observer (inclination $i$ ), and the flow direction (angle $\varphi$ away from the disk axis; see Eq. (2)). The spherical triangle shaded in gray is used to calculate the angle between the flow direction and the line of sight.

spectrum. Which model applies to DG Tauri needs more detailed multi-wavelength observations and comprehensive thermochemical disk opacity modeling.

The ${ }^{13} \mathrm{CO}$ intensity map (Fig. B.1-bottom) is similar, showing bright emission mostly in the 0,2 and $00^{\prime \prime} 6$ radius range. In addition however, as already noticed in the first-moment map (Fig. 1b), a diagonal band from NE to SW suppresses emission along the projected minor axis of the disk, mostly coinciding with an area for which a Keplerian disk shows low radial velocities. This symmetric feature is very likely related to the radial velocity relative to the position of the observer and seems to reach across the disk diameter. No bright highvelocity emission is evident in the map (very different from ${ }^{12} \mathrm{CO}$ in Figs. $2 \mathrm{c}, \mathrm{d}$ ), indicating that like for $\mathrm{C}^{18} \mathrm{O}$, the inner-disk emission is relatively faint. We conclude that the ${ }^{13} \mathrm{CO}$ emission from most likely the entire disk surface is absorbed by a foreground layer of cool gas at specific, small velocities relative to $v_{\text {sys }}$.

\section{Appendix C: Flow geometry}

Figure C.1 shows the adopted geometry of the star-disk-jet system of DG Tauri together with the geometry of the flow relative to the axis and the line of sight. The left half of the diagram explains the geometry of projection of the blueshifted flow emission onto the plane of the disk (Eq. (1)). We assume a flow emitting at a height $z$ above the disk midplane. The half-sphere on the right describes the geometry of the flow and defines angles between the disk rotation axis, the line of sight, and the flow direction at the position of the sphere center, at a height $z$ above the disk plane at azimuth $\alpha$. Here, we assume an axisymmetric flow with an outward inclination angle of $\varphi$ from the local vertical. 